\title{
Strategies of the Brazilian Chronic Kidney Disease Prevention Campaign (2003-2009)
}

\author{
Gianna Mastroianni-Kirsztajn ${ }^{a, b}$ Marcus G. Bastos ${ }^{a, c}$ Emmanuel A. Burdmann ${ }^{a}$ d

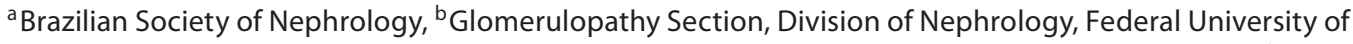 \\ São Paulo, São Paulo, 'Division of Nephrology, Federal University of Juiz de Fora, Juiz de Fora, and d Division of \\ Nephrology, University of São Paulo, São Paulo, Brazil
}

\section{Key Words}

Prevention $\cdot$ Campaign $\cdot$ Proteinuria $\cdot$ Creatinine $\cdot$ Chronic kidney disease $\cdot$ End-stage renal disease

\begin{abstract}
In Brazil, as in the rest of the world, the prevalence of chronic kidney disease (CKD) is increasing. In order to alert the population, health professionals and authorities to this risk, in 2003, the Brazilian Society of Nephrology launched a CKD prevention campaign called 'Previna-se'. In addition, since its onset, Brazil has participated in the World Kidney Day efforts and has developed several prevention strategies. Here, we summarize the main strategies adopted in this campaign (population screening, events and meetings, distribution of educational materials, routine report of estimated glomerular filtration rate) and our initial results, sharing practical experience that could be useful in other developing countries.
\end{abstract}

Copyright ๑ 2010 S. Karger AG, Base

\section{Introduction}

More than 1 million people are alive due to renal replacement therapy, and $90 \%$ of them are in developed countries [1]. On the other hand, the prevalence of chronic kidney disease (CKD) is considered higher in develop- ing countries than in the developed world [2]. Considering the current global burden of CKD, this suggests that many people are probably dying all over the world, and especially in poor countries, due to renal failure without receiving adequate treatment or even a diagnosis.

Worldwide data about the prevalence and incidence of $\mathrm{CKD}$ are scarce, and the great majority of figures correspond to information on patients who started renal replacement therapy. The situation is similar in Brazil. According to the 2008 census of the Brazilian Society of Nephrology (Sociedade Brasileira de Nefrologia), the estimated number of patients on chronic dialysis was 87,044 [3], and similar to the rest of the world, this number is increasing (fig. 1).

Unfortunately, CKD is not sufficiently recognized by the population and not adequately diagnosed in most places in Brazil. Due to this alarming increase in prevalence and underdiagnosis, in 2003 the Brazilian Society of Nephrology initiated a group to deal with CKD prevention. This team developed a national prevention campaign called 'Previna-se' (literally 'Prevent Yourself'). The name of this campaign infers that people should 'prevent' health problems themselves - in this case, renal diseases. In fact, it sounds like an invitation to prevent CKD.

The main objectives of Previna-se were to alert the population, health professionals and authorities to the renal disease problem, improve early detection of CKD, fa-

\section{KARGER}

Fax +41613061234 E-Mail karger@karger.ch www.karger.com
(C) 2010 S. Karger AG, Base

$1660-2110 / 11 / 1173-0259 \$ 38.00 / 0$

Accessible online at:

www.karger.com/nec
Gianna Mastroianni-Kirsztajn

Glomerulopathy Section, Division of Nephrology, UNIFESP

Rua Botucatu 740

São Paulo, SP 04023-900 (Brazil)

Tel. +55 115904 1699, Fax +55 115904 1684, E-Mail gianna@ @efro.epm.br 


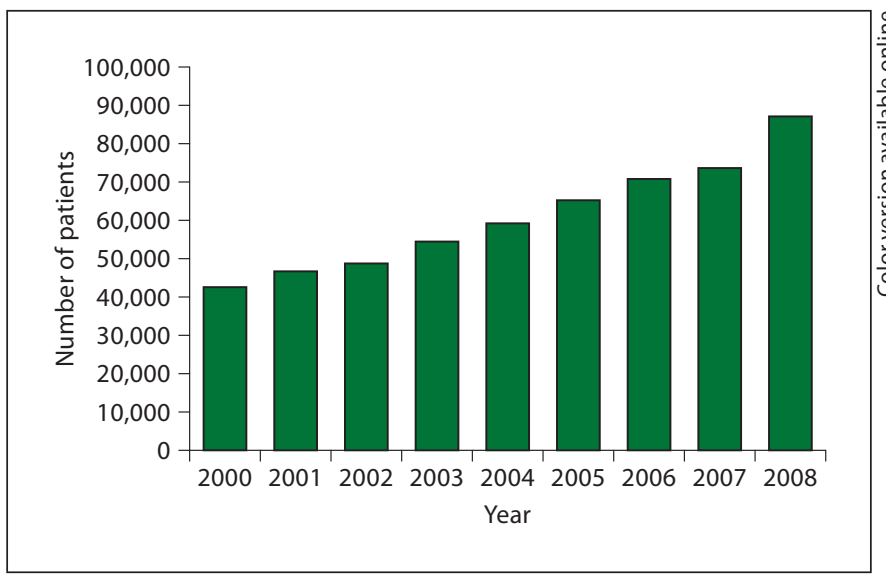

Fig. 1. The annual estimated prevalence of patients on dialysis in Brazil.

cilitate the identification of patients who are at the greatest risk of progression to renal failure, and promote interventions to slow the progression of CKD. In addition, since the onset of Previna-se, Brazil has participated in the World Kidney Day efforts and has developed several prevention strategies.

It is worth noting that models used in underdeveloped or developing countries are not commonly made public. Although this project has been going on for the last 6 years, it is not well known abroad. In order to share this experience, we describe here the strategies used during this Brazilian campaign for CKD prevention.

\section{Strategy 1: Awareness of Nephrologists}

The first step was to guarantee that all nephrologists in the country were aware of the exponential worldwide and local increase in CKD [4], particularly the documented increase in incidence of end-stage renal disease (fig. 1). Since 2003, more than 2,500 specialists in nephrology have received information about this health problem (newsletters, journals, special supplements of the Brazilian Journal of Nephrology), invitation letters to participate in the prevention campaign and free information material created by the prevention committee.

Once this specific group of health professionals recognized the importance of the problem, they responded to the call of our prevention committee and developed their own local campaigns. Today, all 26 states of Brazil have different sites that participate in the Previna-se campaign.

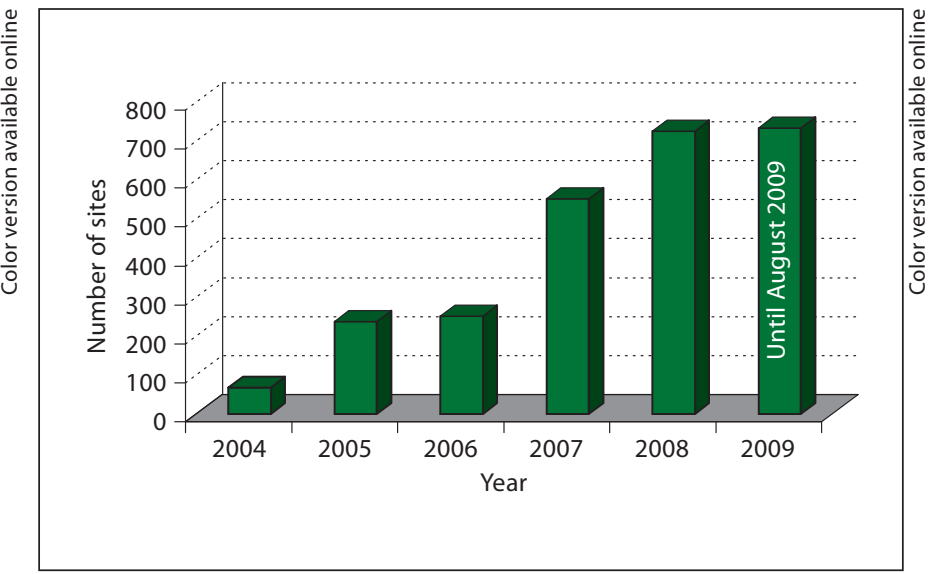

Fig. 2. Number of Brazilian sites involved in the Previna-se campaign since 2004 .

In 2004, in response to the committee's invitation, 68 centers had developed specific programs to educate the public on renal disease and its burden. In the following years, participation rapidly increased, with 236 sites in 2005, 251 in 2006, 551 in 2007 and 725 in 2008 (fig. 2). In 2009 , more than 700 programs were registered at the Brazilian Society of Nephrology again. This is what we call a 'continuous prevention campaign' because every site can develop the campaign any time during the year, contrasting with larger campaigns, which are limited to specific commemorative dates.

It is noteworthy that nephrologists are not the only participants in these public education efforts; other health professionals, teachers, students, patients and their relatives have also volunteered.

In addition, national CKD prevention meetings have been developed annually during the week of the World Kidney Day since 2006, including different preventive approaches directed to nephrologists and other health professionals (scientific lectures), to the community (population screenings) and to the government authorities (political meetings).

\section{Strategy 2: Availability of Educational Material}

The prevention committee produced educational material at the same time that strategy 1 was implemented. The folders, booklets and posters were freely available.

The material included folders with information about renal disease which highlighted that CKD is frequently 


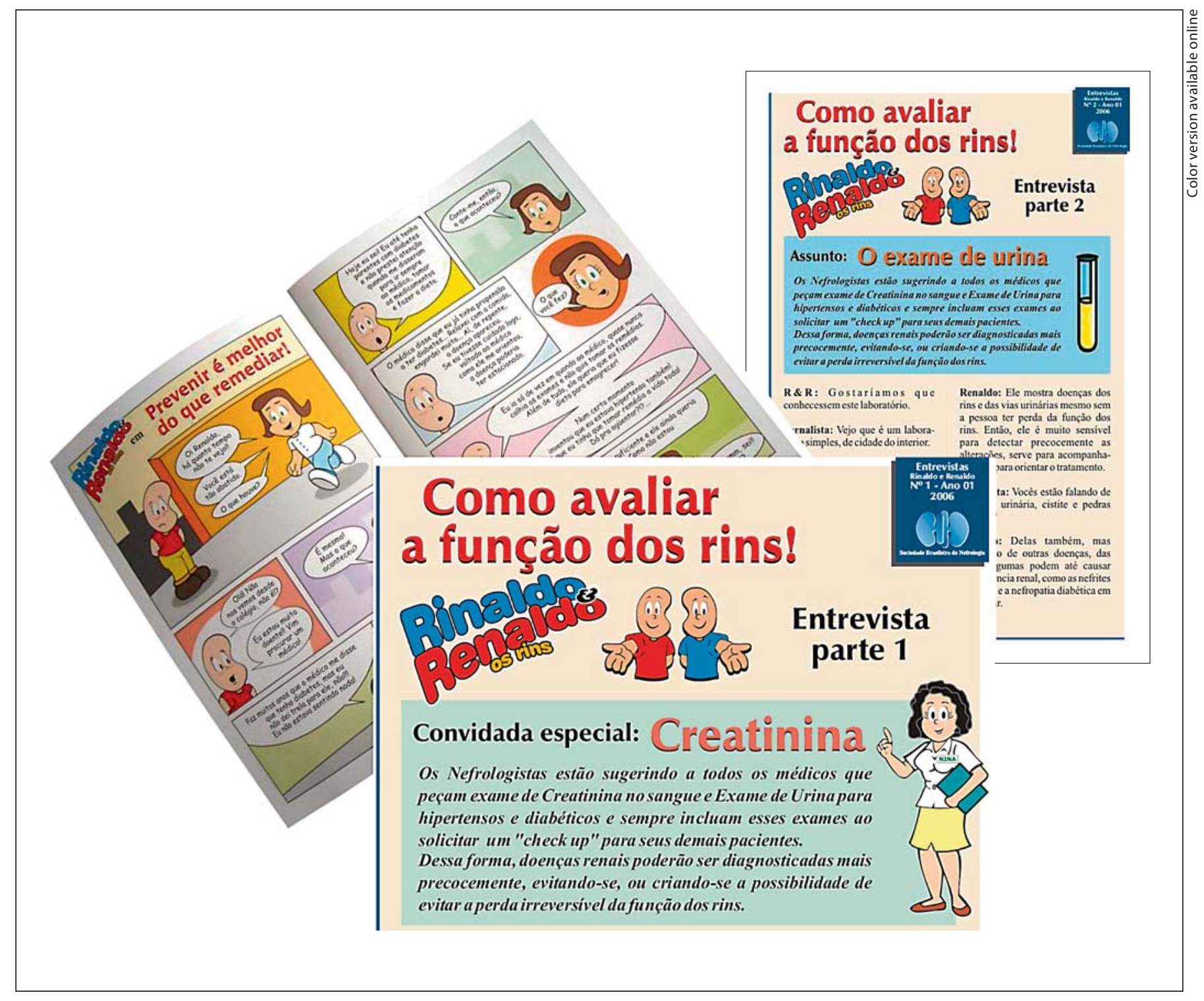

Fig. 3. The booklet tells the history of a kidney-shaped 'diabetic character' that has just been diagnosed with $\mathrm{CKD}$, and the folders contain information for the public about serum creatinine and urinalysis.

asymptomatic, described the main symptoms (when present) and the main renal diseases, recommended what to do and where to find more information. All materials instruct the public to look for a doctor in case of doubt or any suspicious symptom. Folders and booklets also explained the importance of serum creatinine determination and of urinalysis (fig. 3). Currently, more than 3.8 million educational folders have already been distributed all over the country during campaign events.
In addition, buttons and T-shirts with prevention messages were also created, for example: 'I take care of my kidneys. And you?' (a button created in 2003) and 'How is your creatinine?' (T-shirt created in 2004). These materials were designed to be used in field campaigns or in daily work. Other slogans have also been frequently repeated in our folders and talks: 'Renal disease can be silent' or 'You can have a renal disease and not know it. Test your kidneys!. 


\section{Strategy 3: Education of the Brazilian Population}

All media channels were used in order to alert people to the burden of CKD, but this approach by itself was not sufficient to effectively educate the public. Soon, it was clear that performing frequent population screenings and making these screenings public represented a better approach.

In fact, as previously emphasized by Narva [5], 'screening is part of the kidney disease education'. It is worth noting that even if the contribution of general population screenings is questionable from a strictly cost-benefit point of view, their role as an education tool is well established. This motivated us to have field campaigns, which have become increasingly popular in Brazil, as demonstrated by the number of participating sites (fig. 2).

The last strategy we developed was the creation of a film called 'Visiting the World of the Kidneys', which is a cartoon where everybody has a kidney shape; visitors go on a tour and learn about young and old kidneys and the most frequent diseases, causes and symptoms; they also visit primary care units where blood pressure measurements and examinations are performed. This film is freely available for all visitors who want to use it (available at http://www.sbn.org.br/previna.htm).

\section{Strategy 4: Awareness of Other Health Professionals}

Detecting individuals with renal disease and referring them to primary care services or their family physicians would create a new problem if the respective professionals are not aware of the importance of early diagnosis, of their own role in this context and of possible effective treatment measures.

Thus, our prevention group is continuously making contact with primary care physicians and different specialists by several means such as their own journals and congresses, where we share information about the diagnosis and management of CKD, as well as the importance of early referral to nephrologists.

In addition, letters and folders directed at other specialists have emphasized the following suggestion: 'in a check-up you should always include urinalysis and serum creatinine determination'.

Tables showing the estimated glomerular filtration rate (eGFR) according to age, sex and serum creatinine levels were produced for use in primary care services, as well as for training health professionals that directly assist the population [6]. The development of collaborative events with the Brazilian Society of Clinical Pathology and Laboratory Medicine and related publications have contributed to a wider acceptance of laboratory eGFR reporting (strategy 5) in different states of Brazil. This step was essential to the implementation of the next strategy.

\section{Strategy 5: Routine Reports of eGFR}

Although it was not legislatively mandated as in some states of the USA [7], the amount of eGFR reports is increasing all over Brazil. Many laboratories report eGFR for all serum creatinine results. Additionally, some of the laboratories explain (in a note) that the result is presented as a suggestion by the Brazilian Societies of Nephrology and of Clinical Pathology and Laboratory Medicine.

It is remarkable that in Brazil, even before the Previnase campaign initiative, a few laboratories had already reported eGFR together with serum creatinine results to give a Modification of Diet in Renal Disease (MDRD) or Cockcroft-Gault estimation of glomerular filtration. In order to standardize the procedure, the campaign team has had the support of an allied laboratory since 2005, which serves as a model. All other services that want to adopt the eGFR can resolve their doubt by calling the manager of this model laboratory. The leading laboratory is the central laboratory of the Kidney and Hypertension Hospital of the Federal University of São Paulo (Hospital do Rim e Hipertensão, UNIFESP), and the equation used is the MDRD formula [8].

Some laboratories use the Cockcroft-Gault formula, and others calculate eGFR using the MDRD study equation and routinely report eGFR associated with serum creatinine results for patients who are over 16 years old. Because the MDRD estimating equation does not produce accurate results when the eGFR is higher than 60 $\mathrm{ml} / \mathrm{min} / 1.73 \mathrm{~m}^{2}$, according to the recommendation of the NKDEP (National Kidney Disease Education Program) [8], we report a numeric result only when the eGFR is equal or inferior to $60 \mathrm{ml} / \mathrm{min} / 1.73 \mathrm{~m}^{2}$.

Although the eGFR is still not used everywhere in Brazil, nephrologists and clinical pathologists are increasingly aware this is a recommended and easily available method for the evaluation of renal function in everyday practice. Therefore, many of them have looked for information in the aforementioned laboratory. There have not been too many problems in the implementation process because the procedure is adopted voluntarily when the services are adequately prepared for this. In contrast, in 
other sites, laboratories experienced problems in implementing mandatory eGFR reporting, which included software and programming issues, difficulties with older laboratory information systems, and incomplete patient age and gender information that is necessary to calculate and report eGFR [7].

It is of note that the role of routine reporting of eGFR as an isolated screening tool is debated, and it has limitations that must be recognized, as well as the inaccuracy observed at higher levels of glomerular filtration rate. Possibly, such determination alone is less useful for the detection than for the evaluation and management of CKD. Certainly, additional measurements of albuminuria improve the value and precision of early identification of CKD, especially when these determinations are performed in a risk population [9].

\section{Strategy 6: Population Screening}

In several events held in Brazil, screening is performed using dipsticks and, less frequently, serum creatinine determination. The prevention team contacts nephrologists to promote these events, to measure blood pressure and to analyze urine by using dipsticks. In addition, due to the high prevalence of diabetes as a cause of CKD stage 5 , blood glucose is also tested. The use of a standardized questionnaire to collect data (available on the Brazilian Society of Nephrology homepage and in the Brazilian Journal of Nephrology supplement Proposals for Prevention of CKD in Brazil) was also indicated [10].

It is necessary to make clear that in the population screening campaigns here described, an initial test (usually dipstick for detection of proteinuria) is performed, and the individuals with altered results are referred to medical services (primary care units or a specific outpatient service that gives support to that event) with a recommendation to be clinically evaluated and at least repeat urinalysis. They are not considered as patients with CKD during the campaign, but only after being submitted to complete clinical evaluation and complementary examinations to confirm the suspicion that has been based on screening information, blood pressure measure and/or altered urine examination results and/or serum creatinine values.

Although it is difficult to integrate information in a continental country like Brazil, the prevention team asked volunteers to report their experience and results, as well as to send photographs of the events. Most of this work is documented on our homepage (available at http:// www.sbn.org.br/previna.htm), including data from population screenings.

As an example, we here present the results of two days of screening performed by the Previna-se team in a public park (accessible to all social levels) in São Paulo, the biggest Brazilian city, which is localized in the southeast of the country. Those screening campaigns involved 758 subjects: $53 \%$ were females, most of them adults (31\% were $20-39$ years old and $47 \%$ were $40-59$ years old), $53 \%$ had hypertension and $7.8 \%$ had diabetes. At the time of screening, $20 \%$ had blood pressure levels equal to or higher than $140 / 90 \mathrm{~mm} \mathrm{Hg}$, and $7.5 \%$ tested positive for proteinuria. Among the cases of proteinuria, $47 \%$ were individuals that reported to have hypertension $(28 \%$ with high levels at the screening); $10.5 \%$ had cardiovascular disease; $53 \%$ were between 40 and 59 years old, and 3.5\% presented associated hematuria. In a population present in a public park and interested in information about renal disease, we found that proteinuria was common, and $20 \%$ of the individuals had inadequate blood pressure levels, even those already being treated for hypertension. Similarly, in the evaluation of 5,992 subjects in public places performed by the São Paulo Society of Nephrology (SONESP), proteinuria was documented in $7.1 \%$ of the cases. Indeed, accurate data about the frequency of the early stages of CKD in Brazil and Latin America are still scarce $[1,11]$ and current campaign screenings make an initial contribution to understanding this disease profile in our population.

It is known that screening is more effective when directed at groups at a higher risk of developing CKD; however, the members of the prevention team believe that such 'screening' campaigns in public places are important to alert the population and health professionals about the risks of CKD and their epidemic behavior, and also for early referral.

\section{Strategy 7: Detection Followed by Adequate Care}

Undoubtedly, early detection of CKD and adequate assistance in primary care units are ideal for prevention efforts to become effective. Although these measures are still incipient because they are not yet an official governmental policy in Brazil, they have already been effective in some cities. Eventually, government representatives will respond to the requests from nephrologists, creating assistance conditions for the follow-up of the recently identified individuals with CKD (for example, in Mogi das Cruzes, state of São Paulo, a group of nephrologists 
have been using a renal health model since 1990, which has been adopted by the local health authorities). However, university nephrology services are the main source of assistance for these recently identified patients as part of their own prevention and research programs. Additionally, medical students form special groups to study CKD and are an important link between the community and nephrologists [12].

Although its role is still debated, as cited above, population screening by itself is increasingly being recognized as an important public health tool for promoting the early identification of people who are at risk of progressive CKD. It is also an opportunity to educate the public, patients, nonnephrologist health professionals and health care providers on the risks of CKD and the benefits of early care.

In addition, once the medical care system becomes responsible for patients that have been identified in population screenings all over the country, we expect that the natural history of CKD in Brazil will change, similar to what occurred in Japan, where a successful screening program associated with full medical assistance has been developed over the last four decades [13].

\section{Final Comments}

Public education possibly achieves the best cost-benefit ratio to prevent CKD in developing countries. Additionally, many nephrologists are responsible for the education of future health professionals that are currently graduating at their universities. Teachers talk about prevention in their nephrology classes, and they encourage students to participate in prevention campaigns. Few centers are involved in this initiative, but it is possibly one of the most solid actions directed at the development of preventive medicine. Moreover, training nonnephrologist physicians to cope with the increasing number of CKD patients, as well as to recognize the importance of early referral for the timely intervention and management of CKD, is absolutely essential to the success of a CKD prevention program.

The Brazilian prevention effort is still evolving, but since 2003, our nephrologists have discussed this theme more frequently, looked for solutions together and demonstrated that prevention of CKD is an extremely important goal for nephrology. Now we can say that the Previna-se program was at least able to decrease unawareness regarding CKD in our country, a first step on the long way to reach an effective prevention of this disease.

\section{Acknowledgments}

We are grateful to all volunteers and also to the secretary and directory members of the Brazilian Society of Nephrology that have worked on each campaign since 2003. G.M.-K. and E.A.B. are partially supported by CNPq (National Council for Research and Development), Brazil.

\section{References}

1 Perico N, Plata R, Anabaya A, Codreanu I, Schieppati A, Ruggenenti P, Remuzzi G: Strategies for national health care systems in emerging countries: the case of screening and prevention of renal disease progression in Bolivia. Kidney Int Suppl 2005;97:S87S94.

$\checkmark 2$ Alebiosu CO, Ayodele OE: The global burden of chronic kidney disease and the way forward. Ethn Dis 2005;15:418-423.

3 Sesso R, Lopes AA, Thomé FS: Brazilian dialysis census report, 2008. Braz J Nephrol 2008;30:233-238.

4 Hamer RA, El Nahas AM: The burden of chronic kidney disease. BMJ 2006;332:563564 .
5 Narva A: Screening is part of kidney disease education. Clin J Am Soc Nephrol 2007;2: 1352-1354.

6 Bastos MG: Practical method for evaluation of glomerular filtration rate. Braz J Nephrol 2006;28(suppl 1):21-24

7 McDonough DP: New Jersey's experience: mandatory estimated glomerular filtration rate reporting. Clin J Am Soc Nephrol 2007; 2:1355-1359.

8 Moreira SR, Mastroianni Kirsztajn G: Estimated creatinine clearance in the laboratory routine. Braz J Nephrol 2006;28(suppl 1):2527.

-9 Stevens LA, Coresh J, Greene T, Levey AS: Assessing kidney function: measured and estimated glomerular filtration rate. $\mathrm{N}$ Engl J Med 2006;354:2473-2483.
10 Mastroianni Kirsztajn G, Bastos MG: Suggestion of standardization of a screening program for chronic kidney disease. Braz J Nephrol 2007;29(suppl 1):18-22.

-11 Cusumano AM, González Bedat MC: Chronic kidney disease in Latin America: time to improve screening and detection. Clin J Am Soc Nephrol 2008;3:594-600.

12 Bastos MG, Andrade CR, Salgado IAS: The role of the Student Coalition of Support to Nephrology in the prevention of chronic kidney disease. Braz J Nephrol 2007;29(suppl 1):28-31.

$>13$ Imai E, Yamagata K, Iseki K, Iso H, Horio M, Mkino H, Hishida A, Matsuo S: Kidney disease screening program in Japan: history, outcome, and perspectives. Clin J Am Soc Nephrol 2007;2:1360-1366. 


\section{Editorial Comment}

M. El Nahas, Sheffield

Mastroianni-Kirsztajn, Bastos and Burdmann report on the Previna-se program, underway in Brazil for the last few years. This program is an ambitious effort to increase awareness of CKD amongst the public and health professionals. It also includes population screening for CKD. Unfortunately, in the absence of government commitment, the impact of such a program on Brazilian renal healthcare remains somewhat unclear. It is even unclear whether a systematic and in-depth evaluation of its impact on CKD incidence and associated morbidity and mortality has been undertaken.

The Previna-se program, like so many others, raises many issues related to the generous and enthusiastic embracing by the global nephrology community of the 'CKD epidemic' mantra. Many such programs have sprung up all over the world. Many questions remain unanswered:
(1) Are such CKD detection programs well designed if they only test once?

(2) Are they bound to detect a majority of elderly individuals with age-related, decreased, calculated GFR?

(3) How are those tested and found positive followed up?

(4) Does the early detection of CKD1, urinary abnormalities such as microalbuminuria and maintained kidney function, warrant intervention?

(5) Is early intervention effective in a majority of nonprogressive CKD?

(6) Does a reduction in microalbuminuria reduce the associated cardiovascular disease?

(7)Finally, are such programs cost effective?

It is hoped that in the future, plans are in place from the outset to provide some answers to some of these questions, thus justifying the considerable efforts and costs. 